\title{
光/铜共催化远程 $\mathrm{C}-\mathrm{H}$ 键的不对称氰基化反应
}

\author{
成忠明陈品红刘国生* \\ (金属有机化学国家重点实验室和沪港化学合成联合实验室 分子合成卓越中心 \\ 中国科学院上海有机化学研究所 中国科学院大学 上海 200032)
}

\begin{abstract}
摘要 以 $N$-烷氧基邻苯二甲酰亚胺为底物, 利用光与铜共催化, 在蓝光照射下, 实现了远程 $\mathrm{C}-\mathrm{H}$ 键不对称氰基化反 应. 反应中涉及氧自由基的 1,5-氢迁移过程，结合铜催化自由基接力的策略，以良好到优秀的对映选择性、单一的区域 选择性得到了芳基取代的烷基醇类衍生物 $\delta$-位 $\mathrm{C}-\mathrm{H}$ 键不对称㲵基化产物. 该方法条件温和, 具有较好的官能团兼容 性以及底物普适性, 为光学活性的 $\delta$-氧基醇类化合物的合成提供了高效方法.
\end{abstract}

关键词 不对称氰基化; $\mathrm{C}-\mathrm{H}$ 键; 铜催化; 光催化; 1,5 -氢迁移

\section{Enantioselective Cyanation of Remote $\mathrm{C}-\mathrm{H}$ Bonds via Cooperative Photoredox and Copper Catalysis}

\author{
Cheng, Zhongming Chen, Pinhong Liu, Guosheng*
}

(State Key Laboratory of Organometallic Chemistry, and Shanghai Hongkong Joint Laboratory in Chemical Synthesis, Center for Excellence in Molecular Synthesis, Shanghai Institute of Organic Chemistry, University of Chinese Academy of Sciences, Chinese Academy of Sciences, 345 Lingling Road, Shanghai 200032)

\begin{abstract}
Optically pure alkylnitriles are important structural motifs found in agrochemicals, pharmaceuticals, and natural products, which can be further transferred to acids, amines and amides. Direct asymmetric cyanation of $\mathrm{sp}^{3} \mathrm{C}-\mathrm{H}$ bonds represents the most efficient synthetic pathway to these optically pure alkylnitriles. However, selective functionalization of $\mathrm{sp}^{3}$ $\mathrm{C}-\mathrm{H}$ bonds remains a crucial issue due to the inertness of $\mathrm{sp}^{3} \mathrm{C}-\mathrm{H}$ bonds as well as the difficulties in the control of stereoand regioselectivity. Inspired by enzymatic oxygenases and halogenases, such as cytochrome P450 and nonheme iron enzymes, the radical-based $\mathrm{C}-\mathrm{H}$ functionalization has received much attention, which was initiated with a hydrogen atom transfer (HAT) process. Recently, numerous reports have been disclosed for the highly efficient functionalization of $\mathrm{C}-\mathrm{H}$ bonds with an intramolecular HAT process as a key step to govern the reactivity and site selectivity. Our group has developed a copper-catalyzed radical relay process for the enantioselective cyanation and arylation of benzylic $\mathrm{C}-\mathrm{H}$ bonds using TMSCN and $\operatorname{ArB}(\mathrm{OH})_{2}$ as nucleophiles respectively. Mechanistic studies indicated that a benzylic radical generated via a radical replay process can be trapped by a reactive chiral copper(II) cyanide enantioselectively, delivering optically pure benzyl nitriles efficiently. Herein, we communicate the catalytic asymmetric cyanation of remote $\mathrm{C}-\mathrm{H}$ bonds by merging photoredox catalysis with copper catalysis. This reaction proceeds under mild reaction conditions and exhibits good functional group compatibility as well as wide substrates scope. Additionally, the nitrile group was further reduced to amide under hydrogen atmosphere. This reaction provides an efficient pathway to synthesize chiral $\delta$-cyano alcohols and 1,6-amino alcohols. The general procedure is as following: in a dried sealed tube, substrate $\mathbf{1}(0.2 \mathrm{mmol}, 1.0$ equiv. $), \mathrm{Cu}\left(\mathrm{CH}_{3} \mathrm{CN}\right)_{4} \mathrm{PF}_{6}(0.01$ mmol, $5 \mathrm{~mol} \%)$, L $(0.015 \mathrm{mmol}, 7.5 \mathrm{~mol} \%)$ and $\operatorname{Ir}(\mathrm{ppy})_{3}(0.002 \mathrm{mmol}, 1 \mathrm{~mol} \%)$ were dissolved in dichloromethane $(4.0 \mathrm{~mL})$ under $\mathrm{N}_{2}$ atmosphere, and stirred for $30 \mathrm{~min}$. Then, TMSCN (50 $\mu \mathrm{L}, 0.4 \mathrm{mmol}, 2$ equiv.) was added slowly under $\mathrm{N}_{2}$ atmosphere. The tube was sealed with a Teflon-lined cap, and the mixture was stirred under the irradiation of blue LED for 1 $7 \mathrm{~d}$. The reaction mixture was diluted with dichloromethane, filtered through a short pad of celite. A solution of TBAF (3 equiv.) and HOAc ( 3 equiv.) was added to the filtration. The mixture was stirred for $5 \mathrm{~min}$ and then washed with water $(3 \times 10 \mathrm{~mL})$ and dried over anhydrous $\mathrm{Na}_{2} \mathrm{SO}_{4}$. After filtration and concentration, the residue was purified by silica gel chromatography (eluent: petroleum ether/ethyl acetate) to afford target product.
\end{abstract}

Keywords enantioselective cyanation; $\mathrm{C}-\mathrm{H}$ bonds; copper catalysis; photoredox catalysis; 1,5-hydrogen atom transfer

\footnotetext{
*E-mail: gliu@mail.sioc.ac.cn; Tel.: 021-54925346

Received July 4, 2019; published August 15, 2019.

Supporting information for this article is available free of charge via the Internet at http://sioc-journal.cn.

Project supported by the National Basic Research Program of China (No. 973-2015CB856600), the National Natural Science Foundation of China (Nos. 21532009, 21790330 and 21821002), the Science and Technology Commission of Shanghai Municipality (Nos. 17XD1404500, 17QA1405200 and 17JC1401200), and the Key Research Program of Frontier Science (No. QYZDJSSWSLH055) of the Chinese Academy of Sciences.

项目受国家重点基础研究发展计划(973 计划)(No. 973-2015CB856600)、国家自然科学基金(Nos. 21532009, 21790330, 21821002)、上海市科委(Nos. 17XD1404500, 17QA1405200, 17JC1401200)和中国科学院前沿重点项目(No. QYZDJSSWSLH055)资助.
} 


\section{1 引言}

光学活性的烷基腈类化合物广泛存在于天然产物、 药物、农药、材料等领域 ${ }^{[1]}$, 而且可以进一步直接被转 化为合成化学中应用广泛的光学活性的羧酸、酰胺、烷 基胺等 ${ }^{[2]}$. 因此, 光学活性的烷基腈类化合物的高效合 成方法的建立已成为有机化学的研究热点之一. 其中, $\mathrm{C}-\mathrm{H}$ 键的直接不对称氰基化无疑是合成手性烷基腈的 最简洁高效的方式.

$\mathrm{sp}^{3} \mathrm{C}-\mathrm{H}$ 键的选择性官能化反应是有机化学研究 的重要领域之一, 为药物活性分子的后期修饰提供了高 效工具 ${ }^{[3]}$. 但是目前仍然存在着很多挑战, 如 $\mathrm{sp}^{3} \mathrm{C}-\mathrm{H}$ 键往往是比较惰性的, 而且底物分子中的性质相似的 $\mathrm{C}-\mathrm{H}$ 键很多, 很难有效区别, 因此对反应的区域和立 体选择性控制非常困难 ${ }^{[4]}$. 受细胞色素 P450 和非血红 素铁酶等酶催化反应的启发, 国内外很多课题组通过自 由基算氢(HAT)方式发展了不同类型的 $\mathrm{C}-\mathrm{H}$ 键官能化 反应 ${ }^{[5]}$, 尤其是通过动力学有利的 1,5 -氢迁移方式, 高 区域选择性地实现远端 $\mathrm{C}-\mathrm{H}$ 键的官能化反应(图 1a $)^{[6 \sim 9]}$. 这类反应通常涉及如下过程: 生成自由基前体, 产生自由基，区域选择性氢迁移和捕获接力的自由基. 其中, 较常见的引发氢迁移的自由基包括氮自由基、氧 自由基和碳自由基. 2015 年, Muñiz 课题组 ${ }^{[7 a]}$ 报道了碘 催化的 Hofmann-Löffler 反应, 高效合成了含氮杂环化 合物. Knowles 课题组 ${ }^{[7 b]}$ 和 Rovis 课题组 ${ }^{[7 c]}$ 分别利用光 催化氧化 $\mathrm{N}-\mathrm{H}$ 键产生氮自由基, 通过 1,5-氢迁移实现 了远程 $\mathrm{C}-\mathrm{H}$ 键的烷基化反应. 此外, 除了氮自由基驱 动的氢迁移反应, 氧自由基也可以驱动这一反应. 陈以 旳课题组 ${ }^{[\mathrm{b}]}$ 报道了首例可见光催化产生烷氧自由基, 通过 1,5-氢迁移实现 $\mathrm{C}-\mathrm{H}$ 键烯丙基化反应. 焦宁课题 组 ${ }^{[8 \mathrm{e}]}$ 利用银/过硫酸钾体系产生氧自由基, 实现了非活 化烷基醇的直接远程 $\mathrm{C}-\mathrm{H}$ 键官能化反应. 朱晨课题 组 ${ }^{[8 \mathrm{f}-8 \mathrm{~h}]}$ 利用高价碘试剂氧化醇获得氧自由基, 通过 1,5氢迁移实现了 $\delta$ 位芳基化、氧基化等官能化反应. 最近, 祝介平课题组 ${ }^{[8 \mathrm{k}]}$ 光催化还原 $\mathrm{N}-\mathrm{O}$ 键产生氧自由基, 发 生 1,5-氢迁移后, 通过铜催化自由基接力的策略, 实现 了远程 $\mathrm{C}-\mathrm{H}$ 键的叠氮化、氧基化等反应. 相比之下, 碳 自由基驱动的氢迁移反应报道较少, 这是由于不同 $\mathrm{C}-\mathrm{H}$ 键的键能相近, 氢迁移的驱动力较小. 刘心元课 题组 ${ }^{[9]}$ 利用三氟甲基自由基对烯烃加成产生的碳自由 基, 促进 5 位活性氢(胺基 $\alpha$ 位)迁移, 实现了膦催化的胺 基 $\beta$ 位官能化反应. 许鹏飞课题组 ${ }^{[9 c]}$ 报道了光催化芳基 碘化物产生芳基自由基发生 1,5-氢迁移合成氧化吲哚. 尽管通过 1,5 -氢迁移反应可以很好控制反应的区域选择 性, 但是产生的新的烷基自由基往往经历的是直接的自 由基官能化反应, 其对映选择性控制非常困难, 因此相 应的不对称反应几乎没有报道 ${ }^{[8 \mathrm{k}]}$.

我们课题组一直致力于发展自由基不对称反应，通 过发展铜催化自由基接力的策略 ${ }^{[10]}$, 分别以三甲基腈 (a) Remote $\mathrm{C}-\mathrm{H}$ functionalization via HAT

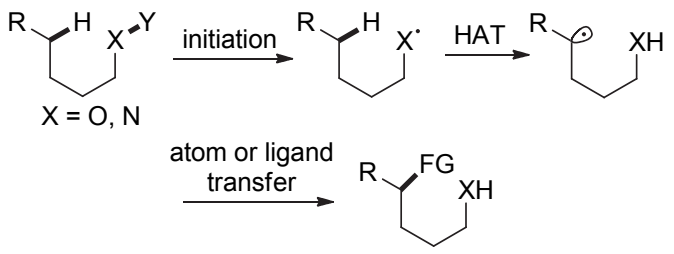

(b) Radical relay process

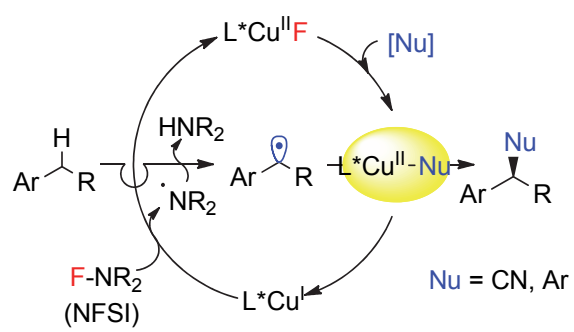

(c) Enantioselective cyanation of remote $\mathrm{C}-\mathrm{H}$ bonds (This work)

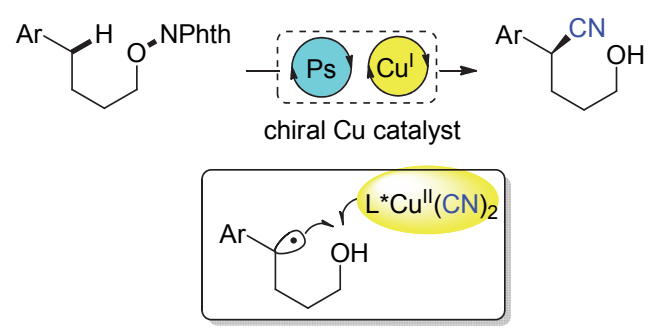

图 1 远程 $\mathrm{C}-\mathrm{H}$ 键官能化反应

Figure 1 Remote $\mathrm{C}-\mathrm{H}$ functionalization via HAT

硅烷(TMSCN)和 $\mathrm{ArB}(\mathrm{OH})_{2}$ 作为亲核试剂, 实现了芐位 $\mathrm{C}-\mathrm{H}$ 键的不对称氰基化和芳基化反应(图 $1 b)^{[11]}$. 机理 研究表明, 通过自由基算氢产生的茮位自由基可以高选 择性地被活性的手性二价铜氧物种捕获, 形成的三价铜 中间体发生还原消除，从而高效得到光学纯的茮基腈类 化合物. 基于这一策略, 进一步实现了一系列铜催化烯 烃的不对称氰基化、芳基化和炔基化反应 ${ }^{[12]}$. 近期, 我 们还报道了光/铜共催化 $N$-羟基邻苯二甲酰亚胺羧酸酯 的不对称脱羧氰基化反应，反应中光催化剂可以还原 $\mathrm{N}-\mathrm{O}$ 键产生氧自由基, 继而脱羧产生苄位自由基, 实 现不对称氰基化反应 ${ }^{[13]}$. 在此基础上，本文利用光/铜 共催化体系, 通过 $N$-烷氧基邻苯二甲酰亚胺底物的单 电子还原产生氧自由基, 发生 1,5 -氢迁移得到芐位自由 基, 实现了高选择性的远程 $\mathrm{sp}^{3} \mathrm{C}-\mathrm{H}$ 键的不对称氰基 化反应(图 1c).

\section{2 结果与讨论}

\section{1 反应条件优化}

文献报道 $N$-烷氧基邻苯二甲酰亚胺是高效产生氧 自由基的活性前体之一 ${ }^{[14,8 b]}$. 在前期工作中我们发现, $\operatorname{Ir}(\mathrm{ppy})_{3}$ 在光照下激发后可以经历氧化淬灭还原 $\mathrm{N}-\mathrm{O}$ 键, 同时产生的 $\operatorname{Ir}(\mathrm{ppy})_{3}{ }^{+}$可以氧化一价铜至二价铜, 实 
现茮基羧酸酯的脱羧不对称氰基化反应 ${ }^{[13]}$. 因此, 设想 通过这一体系有可能通过 $\mathrm{N}-\mathrm{O}$ 键断裂产生烷氧自由 基, 结合氧自由基的 1,5-迁移反应获得苠位自由基, 与 手性铜氰物种反应, 实现氧自由基启动的远程 $\mathrm{C}-\mathrm{H}$ 键 不对称氰基化反应. 于是我们以 $N$-烷氧基邻苯二甲酰 亚胺 $1 \mathbf{a}$ 为模板底物, $\mathrm{Cu}\left(\mathrm{CH}_{3} \mathrm{CN}\right)_{4} \mathrm{BF}_{4}$ 为催化剂和 $\mathbf{L 1}$ 为 配体, TMSCN 为氭基来源, 在 DMF 溶剂中首先考察了 一系列光催化剂对反应的影响, 结果如表 1 所示: 当使 用 $\operatorname{Ir}(\mathrm{bpy})_{3}$ 为光敏剂时, 在 $24 \mathrm{~W}$ 的蓝光照射下, 反应可 以以 $31 \%$ 的收率和 $77 \%$ 的 $e e$ 值得到远程 $\mathrm{C}-\mathrm{H}$ 键不对 称氰基化产物 $\left(\mathbf{2} \mathbf{a}+\mathbf{2} \mathbf{a}^{\prime}\right)$, 其中 $\mathbf{2} \mathbf{a}^{\prime}$ 是生成的羟基产物 $\mathbf{2 a}$ 部分与体系中的三甲基硅基反应所致; 同时反应还伴随 着大量烷氧基还原产物 $\mathbf{3 a}$ 和环化副产物 $4 \mathrm{a}$ 的生成(表 1, Entry 1). 当使用其他氧化性更高的光催化剂时, 如 $\operatorname{Ir}(\text { ppy })_{2}$ (dtbbpy)PF $\mathrm{PF}_{6} \quad([\mathrm{Ir}]-1), \quad \operatorname{Ir}\left(\mathrm{dFCF}_{3} \text { ppy }\right)_{2}$ (dtbbpy) $\mathrm{PF}_{6}$ ([Ir]-2)和 $\mathrm{Ru}(\mathrm{bpy})_{3} \mathrm{Cl}_{2} \cdot 6 \mathrm{H}_{2} \mathrm{O}([\mathrm{Ru}])$, 反应效率并没有得 到提升, 但是反应 $e e$ 值没有受到影响(表 1, Entries 2 4). 然而当使用有机光敏剂 Eosin Y 时, 反应无法发生 (表 1, Entry 5). 溶剂考察显示在二氯甲烷和三氟甲苯中 反应能够表现出较优的对映选择性控制 $(85 \% \mathrm{ee})$, 令人 高兴的是在二氯甲烷中反应的收率能够达到 $93 \%$ (表 1, Entries 6 8). 对照实验显示在避光条件下, 反应无法 发生, 而且铜催化剂和光催化剂都在反应中起了至关重 要的作用(表 1, Entries 9 11).

\section{2 底物普适性研究}

通过对反应条件的系统优化, 我们确定了反应的最 优条件: 以 $N$-烷氧基邻苯二甲酰亚胺 1 为底物, $\operatorname{Ir}(\mathrm{bpy})_{3}$ (1 mol\%)为光催化剂, $\mathrm{Cu}\left(\mathrm{CH}_{3} \mathrm{CN}\right)_{4} \mathrm{BF}_{4}(5 \mathrm{~mol} \%)$ 为催化 剂, $\mathbf{L 1}$ (7.5 mol\%)为配体, TMSCN (2 equiv.)为氧基来源, 在二氯甲烷中 $24 \mathrm{~W}$ 蓝光进行光照反应. 我们对底物的 普适性进行了考察(表 2), 实验结果显示一系列不同取 代的 4-芳基-1-丁醇的邻苯二甲酰亚胺衍生物都可以顺 利反应, 并以良好到优秀的产率高选择性地得到目标产 物(2a 20). 各种不同的官能团(如, 卤素、酯基、醚、 嗍酯、三氟甲氧基等)也都能够在反应体系中兼容; 其中 芳基对位无论是吸电子还是供电子取代基的底物 $\mathbf{1 a}$ 1f 均能够以良好至优秀的收率( $80 \%$ ～93\%), 良好的对 映选择性( $69 \%$ \% $00 \%$ ee $)$ 得到目标产物. 值得注意的是, 对位嗍酯取代的底物 $1 \mathrm{~g}$ 也能兼容反应, 并以 $91 \%$ ee 对 映选择性得到产物 $\mathbf{2 g}$, 这为后续的转化反应提供了可 能. 同样间位不同电性取代的底物 $(1 \mathrm{~h} \sim 1 \mathrm{1}, 1 \mathrm{n} \sim 10)$ 也能 够顺利进行反应; 当芳基邻位有小位阻的取代基时 (1m), 反应仍然可以正常进行, 但增大位阻不利于反应. 我们也进一步考察了菜基底物, 无论是 $\alpha$-荎还是 $\beta$-荎取 代的底物, 分别以 $92 \% \sim 90 \%$ 的收率和 $90 \% \sim 89 \%$ 的 $e e$ 值得到产物 $2 p$ 和 $2 q$. 鉴于杂环在生物活性分子修饰和 药物分子设计中具有重要的应用价值, 我们也考察了含 杂环的底物, 其中噻吩类底物能以高达 $95 \%$ ee 对映选
表 1 反应条件优化 ${ }^{a}$

Table 1 Reaction condition optimization
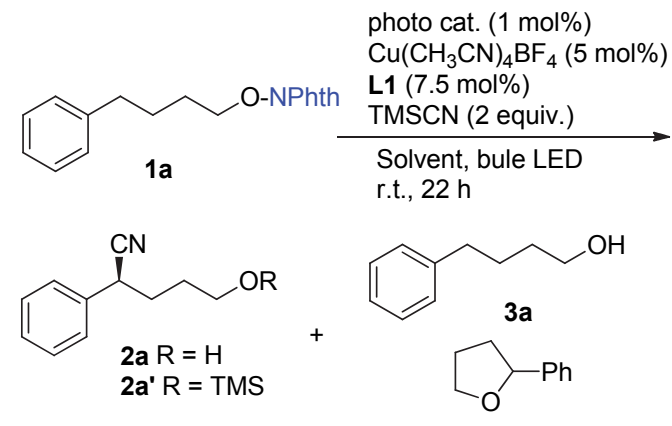

$4 a$

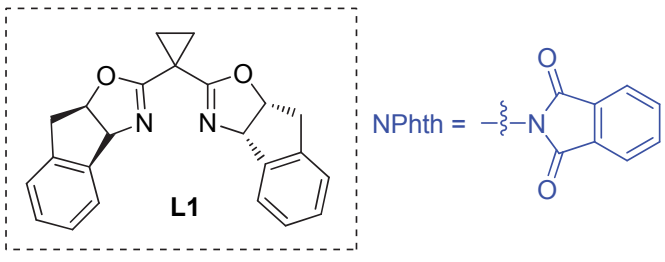

\begin{tabular}{|c|c|c|c|c|}
\hline Entry & Photo cat. & Solvent & $\mathbf{2} \mathbf{a}+\mathbf{2} \mathbf{a}^{\prime}$ Yield $(e e)^{b}$ & $\mathbf{3 a}+\mathbf{4 a}$ Yield $^{b}$ \\
\hline 1 & $\operatorname{Ir}(\mathrm{bpy})_{3}$ & DMF & $31 \%(77 \%)$ & $47 \%$ \\
\hline 2 & {$[\mathrm{Ir}]-1$} & DMF & $16 \%(77 \%)$ & $16 \%$ \\
\hline 3 & {$[\mathrm{Ir}]-2$} & DMF & $30 \%(77 \%)$ & $32 \%$ \\
\hline 4 & {$[\mathrm{Ru}]$} & DMF & $8 \%(76 \%)$ & $11 \%$ \\
\hline 5 & Eosin $Y$ & DMF & 0 & 0 \\
\hline 6 & $\operatorname{Ir}(\mathrm{bpy})_{3}$ & DCM & $93 \%(85 \%)$ & $7 \%$ \\
\hline 7 & $\operatorname{Ir}(\mathrm{bpy})_{3}$ & $\mathrm{PhCF}_{3}$ & $57 \%(85 \%)$ & $6 \%$ \\
\hline 8 & $\operatorname{Ir}(\mathrm{bpy})_{3}$ & $\mathrm{CH}_{3} \mathrm{CN}$ & $71 \%(79 \%)$ & $27 \%$ \\
\hline 9 & $\operatorname{Ir}(\mathrm{bpy})_{3}$ & DCM & 0 & 0 \\
\hline 10 & $\operatorname{Ir}(\text { bpy })_{3}$ & DCM & 0 & 0 \\
\hline 11 & - & DCM & 0 & 0 \\
\hline
\end{tabular}

${ }^{a}$ The reactions were conducted on $0.1 \mathrm{mmol}$ scale with photocatalyst $(1 \mathrm{~mol} \%)$ and $\mathrm{Cu}\left(\mathrm{CH}_{3} \mathrm{CN}\right)_{4} \mathrm{BF}_{4}(5 \mathrm{~mol} \%)$ in solvent $(2 \mathrm{~mL})$ at room temperature, irradiated with $24 \mathrm{~W}$ blue LED. ${ }^{b}$ Crude ${ }^{1} \mathrm{H}$ NMR yield with $\mathrm{CH}_{3} \mathrm{NO}_{2}$ as an internal standard, enantiomeric excess (ee) values were determined by HPLC on a chiral stationary phase. ${ }^{c} \ln$ dark. ${ }^{d}$ Without $\mathrm{Cu}\left(\mathrm{CH}_{3} \mathrm{CN}\right)_{4} \mathrm{BF}_{4} . \quad[\mathrm{Ir}]-1=$

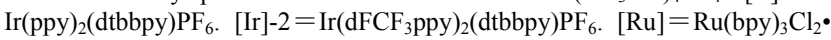
$6 \mathrm{H}_{2} \mathrm{O}$.

择性顺利给出目标产物 2r. 烷基链上的偕二取代也不 会影响 1,5-氢迁移反应, 反应仍然能够以 $85 \%$ 的收率和 $83 \%$ 的 $e e$ 值获得产物 $2 \mathrm{~s}$. 此外 2-乙基-苯甲醇类衍生物 也能顺利进行反应，以 $75 \%$ 的收率和 $80 \%$ 的 $e e$ 值得到产 物 2t. 最后, 所得到的目标产物 $1 \mathrm{a}$ 可以通过简单的兰尼 镍氢化, 高收率地得到光学活性的 5-差基烷基酰胺类化 合物 5, 同时对映选择性得到保留(Eq. 1).

\section{3 反应机理推测}

基于前期的研究工作 ${ }^{[13]}$, 我们推测可能的反应机 理如图 2 所示: 光催化剂 $\operatorname{Ir}(\mathrm{ppy})_{3}$ 在蓝光照下被激发, 激 发态的 $\operatorname{Ir}(\mathrm{III})^{*}$ 具有很强的还原性, 还原底物 $N$-烷氧基 邻苯二甲酰亚胺 1 形成阴离子自由基 int.I, 进一步分解 
表 2 底物范围 ${ }^{a}$

Table 2 Substrate scope

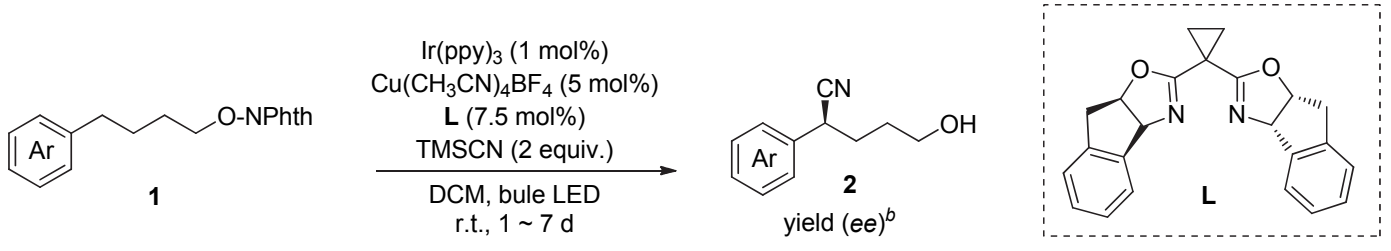<smiles>COC(=O)c1ccc(C(C)CCCO)cc1C(=O)OC(C)(C)C(C)(C)C</smiles>

$93 \%(86 \%$ ee $)$

$85 \%(79 \%$ ee $)$

$85 \%(83 \%$ ee $)$

$84 \%(84 \%$ ee $)$

$86 \%(90 \%$ ee $)$<smiles>COc1ccc(C(C#N)CCCO)cc1</smiles><smiles>COc1cc(OC)cc([C@H](C#N)CCCO)c1</smiles>

85\% $(89 \% \text { ee })^{c}$

$82 \%$ (84\% ee)

$81 \%(92 \%$ ee $)$<smiles>N#CC(CCCO)c1ccc2c(c1)CCC2</smiles>

$79 \%(74 \%$ ee)<smiles>CC(C)(CO)CC(C#N)c1ccccc1</smiles>

$2 \mathrm{~s}$

85\% (83\% ee)<smiles>Cc1c(F)cc(C(C#N)CCCO)cc1F</smiles>

$77 \%$ (91\% ee)

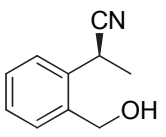

2t

$75 \%(80 \%$ ee $)$

${ }^{a}$ All the reactions were conducted on $0.2 \mathrm{mmol}$ scale. ${ }^{b}$ Isolated yields after treatment with TBAF/HOAc (3 equiv.) and enantiomeric excess (ee) values were determined by HPLC on a chiral stationary phase. ${ }^{c}$ For easy separation, alcohol was converted to ester with AcCl.

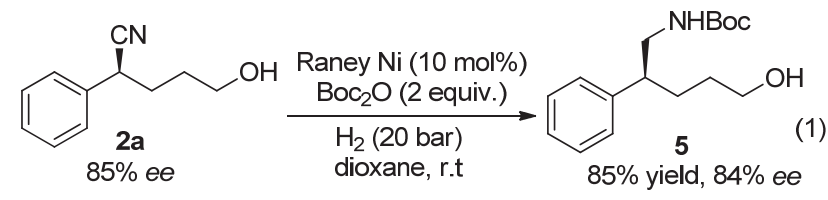

为氧自由基 int.II 和邻苯二甲酰亚胺阴离子 $\left(\mathrm{PhthN}^{-}\right)$. 随 后发生 1,5-氢迁移得到苄位自由基 int.III; 同时被氧化 淬灭后的光催化剂 $\operatorname{Ir}(\mathrm{IV})$ 可以氧化一价铜 $\left(\mathrm{L}^{*} \mathrm{Cu} \mathrm{Cu}^{\mathrm{I}} \mathrm{CN}\right)$ 形 成二价铜 $\left(\mathrm{L}^{*} \mathrm{Cu}^{\mathrm{II}} \mathrm{CN}\right)$, 再生光催化剂 $\mathrm{Ir}(\mathrm{III})$. 二价铜中间 体 $\left(\mathrm{L}^{*} \mathrm{Cu}{ }^{\mathrm{II}} \mathrm{CN}\right)$ 在邻苯二甲酰亚胺阴离子的促进下, 与 TMSCN 反应形成高活性的 $\mathrm{L}^{*} \mathrm{Cu}(\mathrm{CH})_{2}$, 快速地捕捉茮 基自由基 int.III, 高选择性得到远程 $\mathrm{C}-\mathrm{H}$ 键不对称氰 基化产物 2, 同时再生一价铜催化剂. 在整个反应中, 光催化剂的氧化淬灭循环 $(C y c l e A)$ 与铜催化不对称氰 基化反应(Cycle B)的匹配非常关键. 根据前期的脱羧氰 基化反应 ${ }^{[13]}, \operatorname{Ir}(\mathrm{ppy})_{3}{ }^{+}$的还原电势是 $\left\{E_{1 / 2}{ }^{\mathrm{red}}\left[\mathrm{Ir}^{\mathrm{IV}} / \mathrm{Ir}^{\mathrm{III}}\right]=\right.$ $+0.77 \mathrm{~V}$ vs. SCE in $\mathrm{MeCN}\}$, 而手性配体配位一价铜 $\left(\mathrm{L} * \mathrm{Cu}{ }^{\mathrm{I}} \mathrm{CN}\right)$ 的还原电势为 $\left\{E_{1 / 2}{ }^{\mathrm{red}}\left[\mathrm{Cu}^{\mathrm{II}} / \mathrm{Cu}^{\mathrm{I}}\right]=+0.36 \mathrm{~V}\right.$ vs. $\mathrm{SCE}$ in $\mathrm{MeCN}\}$, 因此 $\operatorname{Ir}(\mathrm{ppy})_{3}{ }^{+}$可以氧化一价铜至二价 铜, 促进两个催化循环顺利进行.

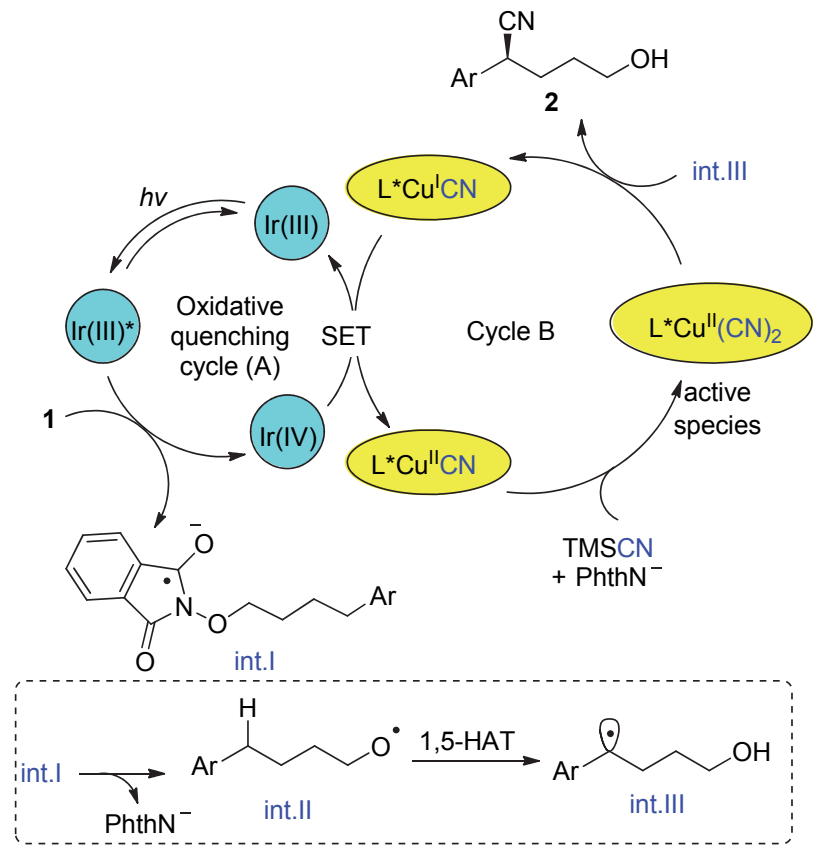

图 2 推测的反应机理

Figure 2 Proposed mechanism 


\section{3 结论}

本文基于铜催化自由基接力的策略, 利用光/铜共 催化, 发展了 $\delta$-芳基烷基醇的远程 $\mathrm{C}-\mathrm{H}$ 键不对称氭基 化反应. 反应以 $N$-烷氧基邻苯二甲酰亚胺为底物, 通过 光照产生氧自由基, 经历 1,5 -氢迁移过程, 以良好到优 秀的对映选择性, 区域单一地得到光学活性的 $\delta$-㲵基醇 类化合物. 该方法条件温和, 具有较好的官能团兼容性 以及底物普适性. 所得到的产物中氰基可以进一步通过 氢化还原为胺基, 为光学活性的 $\delta$-㲵基醇和 1,6-氨基醇 类化合物的合成提供了高效方法.

\section{4 实验部分}

$\mathrm{C}-\mathrm{H}$ 键不对称氰基化反应操作步骤: 在手套箱中, 往干燥的封管中称取底物 1 (0.2 mmol, 1.0 equiv.)、 $\mathrm{Cu}\left(\mathrm{CH}_{3} \mathrm{CN}\right)_{4} \mathrm{PF}_{6}(0.01 \mathrm{mmol}, 5 \mathrm{~mol} \%)$, 手性配体(0.015 $\mathrm{mmol}, 7.5 \mathrm{~mol} \%$ )和光催化剂 $\operatorname{Ir}(\mathrm{ppy})_{3}(0.002 \mathrm{mmol}, 1$ $\mathrm{mol} \%$ ). 取出后氮气保护下加入二氯甲烷 $(4 \mathrm{~mL})$, 避光 下搅拌 $30 \mathrm{~min}$, 使配体和铜催化剂配位. 然后慢慢加入 TMSCN (50 $\mu \mathrm{L}, 0.4 \mathrm{mmol}, 2$ equiv.), 封管后在 $24 \mathrm{~W}$ 蓝 光照射下室温反应 $1 \sim 7 \mathrm{~d}$. 反应结束后, 用二氯甲烷(10 $\mathrm{mL}$ )稀释, 过硅藻土短柱, 滤液加入 TBAF (3 equiv.)和 HOAc (3 equiv.) 搅拌 $5 \mathrm{~min}$, 加入饱和食盐水, 用乙酸乙 酯萃取, 合并有机相用无水硫酸钠干燥, 过滤, 浓缩后, 用硅胶柱层析分离得到产物 2.

\section{References}

[1] (a) Wang, J.; Liu, H. Chin. J. Org. Chem. 2012, 32, 1643. (王江, 柳 红, 有机化学, 2012, 32, 1643.) (b) Fleming, F. F.; Yao, L.; Ravikumar, P. C.; Funk, L.; Shook, B. C. J. Med. Chem. 2010, 53, 7902.

[2] (a) Rappoport, Z. The Chemistry of the Cyano Group, Interscience Publishers, London, 1970. (b) Larock, R. C. Comprehensive Organic Transformations: A Guide to Functional Group Preparation, 2nd ed., Wiley-VCH, Weinheim, 1999, p. 821.

[3] Cernak, T.; Dykstra, K. D.; Tyagarajan, S.; Vachal, P.; Krska, S. W. Chem. Soc. Rev. 2016, 45, 546.

[4] (a) Meunier, B.; de Visser, S. P.; Shaik, S. Chem. Rev. 2004, 104, 3947. (b) Ortiz de Montellano, P. R. Chem. Rev. 2010, 110, 932.

[5] For some reviews, see: (a) Che, C.-M.; Lo, V. K.-Y.; Zhou, C.-Y.; Huang, J.-S. Chem. Soc. Rev. 2011, 40, 1950. (b) Lu, H.; Zhang, X. P. Chem. Soc. Rev. 2011, 40, 1899. (c) Huang, X.; Groves, J. T. Chem. Rev. 2018, 118, 2491. (d) Bietti, M. Angew. Chem., Int. Ed. 2018, 57, 16618. (e) Pei, P.; Zhang, F.; Yi, H.; Lei, A. Acta Chim. Sinica 2017, 75, 15. (裴朋昆, 张凡, 易红, 雷爱文, 化学学报, 2017, 75, 15.)

[6] For some reviews, see: Stateman, L. M.; Nakafuku, K. M.; Nagib, D. A. Synthesis 2018, 50, 1569 .

[7] (a) Martínez, C.; Muñiz, K. Angew. Chem., Int. Ed. 2015, 54, 8287. (b) Choi, G. J.; Zhu, Q.; Miller, D. C.; Gu, C. J.; Knowles, R. R. Nature 2016, 539, 268. (c) Chu, J. C. K.; Rovis, T. Nature 2016, 539, 272. (d) Chen, D.; Chu, J. C. K.; Rovis, T. J. Am. Chem. Soc. 2017, 139, 14897. (e) Wappes, E. A.; Fosu, S. C.; Chopko, T. C.; Nagib, D. A. Angew. Chem., Int. Ed. 2016, 55, 9974. (f) Liu, T.; Myers, M. C.; Yu, J.-Q. Angew. Chem., Int. Ed. 2017, 56, 306. (g) Becker, P.; Duhamel, T.; Stein, C. J.; Reiher, M.; Muñiz, K. Angew. Chem., Int. Ed. 2017, 56, 8004. (h) Li, Z.; Wang, Q.; Zhu, J. Angew. Chem., Int. Ed.
2018, 57, 13288. (i) Jiang, H.; Studer, A. Angew. Chem., Int. Ed. 2018, 57, 1692. (j) Xia, Y.; Wang, L.; Studer, A. Angew. Chem., Int. Ed. 2018, 57, 12940. (k) Dauncey, E. M.; Morcillo, S. P.; Douglas, J. J.; Sheikh, N. S.; Leonori, D. Angew. Chem., Int. Ed. 2018, 57, 744 (1) Morcillo, S. P.; Dauncey, E. M.; Kim, J. H.; Douglas, J. J.; Sheikh, N. S.; Leonori, D. Angew. Chem., Int. Ed. 2018, 57, 12945. (m) Li, C.; Lang, K.; Lu, H.; Hu, Y.; Cui, X.; Wojtas, L.; Zhang, X. P. Angew. Chem., Int. Ed. 2018, 57, 16837. (n) Chen, H.; Guo, L.; Yu, S. Org. Lett. 2018, 20, 6255. (o) Stateman, L. M.; Wappes, E. A.; Nakafuku, K. M.; Edwards, K. M.; Nagib, D. A. Chem. Sci. 2019, 10, 2693. (p) Zhang, Z.; Stateman, L. M.; Nagib, D. A. Chem. Sci. 2019, 10, 1207. (q) Wu, K.; Wang, L.; Colón-Rodríguez, S.; Flechsig, G.-U.; Wang, T. Angew. Chem., Int. Ed. 2019, 58, 1774. (r) Bao, X.; Wang, Q.; Zhu, J. Nature Commun. 2019, 10, 768. (s) Lang, K.; Torker, S.; Wojtas, L.; Zhang, X. P. J. Am. Chem. Soc. 2019, DOI: 10.1021/jacs.9b05850.

[8] (a) Peng, Y.; Lin, J.-S.; Li, L.; Zheng, S.-C.; Xiong, Y.-P.; Zhao, L.-J.; Tan, B.; Liu, X.-Y. Angew. Chem., Int. Ed. 2014, 53, 11890. (b) Zhang, J.; Li, Y.; Zhang, F.; Hu, C.; Chen, Y. Angew. Chem., Int. Ed. 2016, 55, 1872. (c) Wang, C. Y.; Harms, K.; Meggers, E. Angew. Chem., Int. Ed. 2016, 55, 13495. (d) Hu, A.; Guo, J.-J.; Pan, H.; Tang, H.; Gao, Z.; Zuo, Z. J. Am. Chem. Soc. 2018, 140, 1612. (e) Zhu, Y.; Huang, K.; Pan, J.; Qiu, X.; Luo, X.; Qin, Q.; Wei, J.; Wen, X.; Zhang, L.; Jiao, N. Nat. Commun. 2018, 9, 2625. (f) Wu, X.; Zhang, H.; Tang, N.; Wu, Z.; Wang, D.; Ji, M.; Xu, Y.; Wang, M.; Zhu, C. Nat. Commun. 2018, 9, 3343. (g) Wu, X.; Wang, M.; Huan, L.; Wang, Wang, D. J.; Zhu, C. Angew. Chem., Int. Ed. 2018, 57, 1640. (h) Wang, M.; Huang, L.; Zhu, C. Org. Lett. 2019, 21, 821. (i) Kim, I.; Park, B.; Kang, G.; Kim, J.; Jung, H.; Lee, H.; Baik, M.; Hong, S. Angew. Chem., Int. Ed. 2018, 57, 15517. (j) Guan, H.; Sun, S.; Mao, Y.; Chen, L.; Lu, R.; Huang, J.; Liu, L. Angew. Chem. Int. Ed. 2018, 57, 11413. (k) Bao, X.; Wang, Q.; Zhu, J. Angew. Chem. Int. Ed. 2019, 58, 2139.

[9] (a) Yu, P.; Zheng, S.-C.; Yang, N.-Y.; Tan, B.; Liu, X.-Y. Angew. Chem., Int. Ed. 2015, 54, 4041. (b) Cui, X.; Xu, X.; Jin, L.-M.; Wojtasa, L.; Zhang, X. P. Chem. Sci. 2015, 6, 1219. (c) Chen, J.-Q.; Wei, Y.-L.; Xu, G.-Q.; Liang, Y.-M.; Xu, P.-F. Chem. Commun. 2016 52, 6455. (d) Li, T.; Yu, P.; Lin, J.-S.; Zhi, Y.; Liu, X.-Y. Chin. J. Chem. 2016, 34, 490. (e) Li, L.; Ye, L.; Ni, S.-F.; Li, Z.-L.; Chen, S.; Du, Y.-M.; Li, X.-H.; Dang, L.; Liu, X.-Y. Org. Chem. Front. 2017, 4, 2139. (f) Yuan, W.; Zhou. Z.; Gong, L.; Meggers, E. Chem. Commun. 2017, 53, 8964. (g) Li, T.; Yu, P.; Du, Y.-M.; Lin, J.-S.; Zhi, Y.; Liu, X.-Y. J. Fluorine Chem. 2017, 203, 210. (h) Wang, N.; Ye, L.; Li, Z.-L.; Li, L.; Li, Z.; Zhang, H.-X.; Guo, Z.; Gu, Q.-S.; Liu, X.-Y. Org. Chem. Front. 2018, 5, 2810. (i) Chen, J.-Q.; Chang, R.; Lin, J.-B.; Luo, Y.-C.; Xu, P.-F. Org. Lett. 2018, 20, 2395. (j) Wang, Y.; Wen, X.; Cui, X.; Zhang, X. P. J. Am. Chem. Soc. 2018, 140, 4792. (k) Wen, X.; Wang, Y.; Zhang, X. P. Chem. Sci. 2018, 9 , 5082. (1) Wu, S.; Wu, X.; Wang, D.; Zhu, C. Angew. Chem., Int. Ed. 2019, 58, 1499. (m) Chuentragool, P.; Yadagiri, D.; Morita, T.; Sarkar, S.; Parasram, M.; Wang, Y.; Gevorgyan, V. Angew. Chem. Int. Ed. 2019, 58, 1794.

[10] Wang, F.; Chen, P.; Liu, G. Acc. Chem. Res. 2018, 51, 2036.

[11] (a) Zhang, W.; Wang, F.; McCann, S. D.; Wang, D.; Chen, P.; Stahl, S. S.; Liu, G. Science 2016, 353, 1014. (b) Zhang, W.; Wu, L.; Chen, P.; Liu, G. Angew. Chem., Int. Ed. 2019, 58, 6425. (c) Zhang, W.; Chen, P.; Liu, G. J. Am. Chem. Soc. 2017, 139, 7709.

[12] For cyanations, see: (a) Wang, F.; Wang, D.; Wan, X.; Wu, L.; Chen, P.; Liu, G. J. Am. Chem. Soc. 2016, 138, 15547. (b) Wang, D.; Wang, F.; Chen, P.; Lin, Z.; Liu, G. Angew. Chem., Int. Ed. 2017, 56, 2054. (c) Lu, F.-D.; Liu, D.; Zhu, L.; Lu, L.-Q.; Yang, Q.; Zhou, Q.-Q.; Wei, Y.; Lan, Y.; Xiao, W.-J. J. Am. Chem. Soc. 2019, 141, 6167. For arylations, see: (d) Wu, L.; Wang, F.; Wan, X.; Wang, D.; Chen, P.; Liu, G. J. Am. Chem. Soc. 2017, 139, 2904. (e) Wang, D.; Wu, L.; Wang, F.; Wan, X.; Chen, P.; Lin, Z.; Liu, G. J. Am. Chem. Soc. 2017, 139, 6811. For alkynylation, see: (f) Fu, L.; Zhou, S.; Wan, X.; Chen, P.; Liu, P. J. Am. Chem. Soc. 2018, 140, 10965.

[13] Wang, D.; Zhu, N.; Chen, P.; Lin, Z.; Liu, G. J. Am. Chem. Soc. 2017, 139, 15632.

[14] (a) Curran, D. P.; Kim, D.; Liu, H. T.; Shen, W. J. Am. Chem. Soc 1988, 110, 5900. (b) Kim, S.; Lee, T. A.; Song, Y. Synlett 1998, 471. (c) Zlotorzynska, M.; Sammis, G. M. Org. Lett. 2011, 13, 6264. 